\title{
BMJ Open Longitudinal analysis of growth trajectories in young children of Chinese-born immigrant mothers compared with Australian-born mothers living in Victoria, Australia
}

\author{
Kristy A Bolton (D) , ${ }^{1}$ Peter Kremer (D) ,2 Rachel Laws, ${ }^{1,3}$ Karen J Campbell, ${ }^{1,3}$ \\ Miaobing Zheng ${ }^{1,3}$
}

To cite: Bolton KA, Kremer $P$, Laws R, et al. Longitudinal analysis of growth trajectories in young children of Chineseborn immigrant mothers compared with Australianborn mothers living in Victoria, Australia. BMJ Open 2021;11:e041148. doi:10.1136/ bmjopen-2020-041148

- Prepublication history and additional material for this paper is available online. To view these files, please visit the journal online (http://dx.doi.org/10. 1136/bmjopen-2020-041148)

Received 02 June 2020

Revised 14 December 2020

Accepted 22 January 2021

Check for updates

(c) Author(s) (or their employer(s)) 2021. Re-use permitted under CC BY-NC. No commercial re-use. See rights and permissions. Published by BMJ.

${ }^{1}$ Institute for Physical Activity and Nutrition, School of Exercise and Nutrition Sciences, Deakin University, Geelong, Victoria, Australia

${ }^{2}$ Centre for Sport Research, School of Exercise and Nutrition Sciences, Deakin University, Geelong, Victoria, Australia ${ }^{3}$ Centre of Research Excellence in the Early Prevention of Obesity in Childhood, University of Sydney, Sydney, New South Wales, Australia

Correspondence to

Dr Kristy A Bolton;

kristy.bolton@deakin.edu.au

\section{ABSTRACT}

Background Chinese immigrants are the third largest immigrant group in Australia. Little is known about growth trajectories of their offspring when moving to a Western country. The aim was to describe the growth trajectories between birth to 3.5 years in children of Chinese-born immigrant mothers compared with Australian-born mothers living in Victoria, Australia.

Methods Ten nurse measured weights and lengths from birth to 3.5 years were used to examine growth trajectory using linear spline multilevel models. Five knot points were identified at visit 2 (0.5 months), visit 4 (2 months), visit 5 (4.5 months), visit 8 (18 months) and visit 9 (25 months). Results Ethnic disparities in growth trajectories between these two groups were revealed in models adjusted for birth weight, sex and level of socioeconomic disadvantage. Children of Chinese-born compared with Australian-born mothers revealed different growth rates and significant differences in predicted mean body mass index Z score (zBMI) at all time points from birth to 44 months, except for 12 months. Specifically, when compared with children of Australian-born mothers, children of Chinese-born mothers started with lower predicted zBMl from birth until 0.5 months, had a higher ZBMI from 1 to 8 months and a lower zBMl from 12 to 44 months. Early and sharp acceleration of growth was also observed for children of Chinese-born mothers (0.5-2 months) when compared with children of Australian-born mothers (2-18 months).

Conclusion Differences in growth trajectories exist between young children of Chinese-born and Australianborn mothers. Better understanding of these ethnically patterned growth trajectories is important for identifying key opportunities to promote healthy growth in early life.

\section{INTRODUCTION}

Childhood obesity has serious health consequences $^{1}$ and can track into adult life. ${ }^{2}$ In 2017-2018 in Australia, 24.6\% of children aged 2-4 years old were classified as overweight or obese. ${ }^{3}$ The first 1000 days of a child's life have been emphasised to be crucial in developing a child's potential health and

\section{Strengths and limitations of this study}

- Modelling growth trajectory in a large sample size with longitudinal repeated measurements from birth to 3.5 years old.

- Linear spline multilevel modelling methodology was used which allows examination of $\mathrm{zBMI}$ trajectories across childhood while taking into account the different number of visits and measurements over time.

- Spline and knot modelling methodology allows trajectories to be simplified for easy comparison across populations.

- The model was adjusted for key covariates such as child sex, Indigenous status, gestational age, maternal marital and smoking status, delivery method and level of socioeconomic disadvantage; however, maternal age was not included due to the amount of missing data on this variable.

- While the data were drawn from one local government area in Victoria, Australia; which has a high prevalence of Chinese immigrants, these findings may not be generalisable to the wider population.

development over the life course. ${ }^{45}$ Monitoring growth of infants can, therefore, support healthy growth and development of children. ${ }^{6}$ Infant growth trajectories can give an indication of subsequent risk of poor health later in life. ${ }^{7}$ Proposed determinants of growth trajectories and subsequent overweight and obesity include maternal smoking, prepregnancy maternal body mass index (BMI), socioeconomic status, ethnicity and infant feeding practices (eg, early introduction of solids, introduction of infant formula). ${ }^{589}$

Australia is a multicultural country, with $29 \%$ of inhabitants born overseas. ${ }^{10}$ In 2018, Chinese immigrants in Australia comprised $2.6 \%$ of the total population and were the 
second largest immigrant group in Australia. ${ }^{10}$ Ethnic background has been demonstrated to be an important risk factor for overweight and obesity in Australian primary school aged children with Asian, North African, Middle Eastern, Southern, South Eastern and Eastern European backgrounds. ${ }^{11-13}$ Using language spoken most at home to categorise cultural groups; a higher proportion of Asian primary-school aged children were classified as overweight/obese compared with English-speaking children (27.6\% compared with $22.4 \%$, respectively) ${ }^{13} \mathrm{In}$ another Victorian study, $34.8 \%$ of Eastern Asian primary school aged children were found to be overweight/ obese,${ }^{14}$ which is much higher than the national average of $25.2 \%$ children aged $8-11$ years old who were classified as overweight/obese. ${ }^{3}$ It is important to understand what might drive these differences in overweight and obesity prevalence in early life.

Maternal child and health (MCH) nurses are key providers of regular and free child and family wellness checks ${ }^{15}$ in Victoria, Australia. Given the importance of healthy growth for all development, these nurses measure height and weight at each of 10 scheduled visits between birth and 3 years enabling tracking of both failure to thrive and unexpected rapid growth a well-established risk factor for childhood obesity in the first 6 months of life. ${ }^{16-18}$ There is currently no information on longitudinal growth trajectories of infants or young children of Chinese-born immigrants living in Australia. Understanding growth trajectories may help health practitioners to identify children at the highest risk for later overweight and obesity. ${ }^{19}$

The aim of this study was to compare BMI z score (zBMI) growth trajectories from birth to 3.5 years in young children of Chinese-born and Australian-born mothers residing in Australia. This information will inform optimal targeting of interventions aiming to promote healthy growth in this potentially at risk immigrant population.

\section{METHODS}

\section{Patient and public involvement}

Patients were not involved in the design, conduct, reporting or dissemination of this study.

\section{Study setting and participants}

Victoria's universal Maternal and Child Health Service provides ten contacts from birth until school age (birth, 2, 4, 8 weeks; $4,8,12,18,24,42$ months (3.5 years)) to assess child growth and development; with a focus on child and family well-being. ${ }^{20}$ All data collected at each visit were entered by the MCH nurse into an electronic database.

The longitudinal data in the current study were deidentified and exported from $16 \mathrm{MCH}$ centres located in a local government area in Victoria with a high proportion of Chinese-born immigrants (making up 7\% of the local population).$^{21}$ Data related to all children of Chinese-born mothers; along with a random subsample of Australianborn children was extracted by the custodian of the database. Country of birth for mothers was used to determine ethnicity. Mothers were categorised as 'Chinese-born' if they were born in mainland China. Special Administration Regions such as Hong Kong, Macau, Wolong and Taiwan Province were excluded. ${ }^{22}$

\section{Maternal and child demographic factors}

Demographic information collected from the primary caregiver included mother/child date of birth, mother/child country of birth, postcode, marital status of mother, current smoking status of mothers, child Indigenous status, gestational age, child sex and birth delivery method (vaginal, caesarean, other). Postcode was used to determine the level of socioeconomic disadvantage using Socio-Economic Indexes for Areas (SEIFA) ${ }^{23}$ The level of socioeconomic disadvantage was examined by quintile, however, due to low sample size, quintiles were recoded into dichotomous categories based on the spread of the data: 'low/medium' (quintiles 1-4) and 'high' (quintile 5).

\section{Anthropometric measures}

At each of the 10 visits described above, the MCH nurse collected the age of the child at the current visit; measured the child's weight, length/height, head circumference and noted medical history if applicable (eg, immunisation status). Length was measured (to $0.1 \mathrm{~cm}$ ) in recumbent position on a measuring mat until 2 years of age, there after the child was measured standing upright using a portable stadiometer. Z-scores (zBMI) were calculated using the WHO macro in STATA and the WHO growth standards. ${ }^{24}$ For details regarding the number of visits, mean age, weight, height, zBMI of the 10 time points by ethnicity; refer to online supplemental file 1.

\section{Statistical analyses}

For a flow chart of the sample, refer to online supplemental figure 1. A total of 2226 singleton children and mothers were included in the analysis (1082 children of Chinese-born mothers, hereon referred to as Chineseborn; 1144 children of Australian-born mothers, hereon referred to as Australian-born). Cases were excluded if there was a premature delivery ( $<37$ weeks); the mother was born in regions other than mainland China (eg, Hong Kong); if the child was of low birth weight of $<2500 \mathrm{~g}$ as evidence suggests low birth weight babies may have increased risk of poorer development and illness and may grow differently ${ }^{25}$; no zBMI measurement and age difference between measurement occasions were zero or negative. This resulted in a total of 1864 children and their mothers (930 Chinese-born and 934 Australianborn) with complete data on child sex, birth weight and socioeconomic disadvantage.

Descriptive statistics (means, SD, proportions) were used to summarise data for Chinese-born and Australianborn mothers and their children. Differences between ethnic groups (Chinese vs Australian born) were tested 
using $\chi^{2}$ tests or $\mathrm{t}$-tests. To model the longitudinal zBMI growth trajectory in the current study from birth to 3.5 years, linear spline multilevel models were used to construct a series of linear splines joined at knot points, where the direction or the magnitude of growth changed. ${ }^{26}$ This method allows true shapes of growth trajectories to be modelled and overcomes limitations of traditional methods to examine growth trajectory, which include collinearity of repeated measures, measurement requirements (ie, all individuals being the same age when measured, all individuals having complete measurements), bias from missing data, clustering and difficulty with the interpretation of growth coefficients. ${ }^{26}$ This method has been used in other published studies to examine growth trajectories in early childhood, and also by ethnic subgroups. ${ }^{26-29}$

To identify the knot points, both fractional polynomials and lowess curves were used to identify the best fitting curve, from which five knot points, where the direction or magnitude/slope of the growth changed, were identified at: visit 2 ( 2 weeks $/ 0.5$ months), visit 4 (2 months), visit 5 (4.5 months), visit 8 (18 months) and visit 9 (25 months). This resulted in six splines (growth periods): birth to 0.5 months, 0.5-2 months, 2-4.5 months, 4.5-18 months, 18-25 months and 15-44 months. The basic model included repeated measures of zBMI as the dependent variable; six splines as fixed effects; six splines as a level 1 random effect (accounting for correlation between measures) and $\mathrm{MCH}$ centre as a level 2 random effect (accounting for clustering) with an unstructured covariance structure. The intercept and coefficient (slope) of each spline of the fixed part represent zBMI at birth and growth rates for that growth period. Comparison of average observed and predicted zBMI at each time point from the spline model were similar indicating a good model fit (refer to online supplemental file 1 for model fit information).

To explore whether the growth trajectory differed by being a child of Chinese-born or Australian-born mothers, ethnicity was analysed as the principal fixed effect. The interaction between ethnicity and splines were fitted, and the coefficient of each spline represented the mean difference in growth rates between children of Chineseborn and Australian-born mothers in that growth period. Overall two growth trajectory models were constructed, one unadjusted, one adjusted for covariates. The following covariates were considered: Indigenous status, child sex, gestational age, maternal marital status, delivery method, level of SEIFA and smoking status. Pearson correlation revealed no significant relationships among these covariates, they were simultaneously included in the model as covariates.

Multiple imputation by chained equation with 10 datasets was used to impute missing covariates. The percentage missing on these covariates were $1 \%-15 \%$. Estimates from 10 imputed datasets were combined using the 'mi estimate' command. Given the similarity in results, only the adjusted models are presented. All analyses were conducted using Stata V.14.0 (StataCorp) with significance set at $\mathrm{p}<0.05$.

\section{RESULTS}

\section{Sample characteristics}

Child and maternal sample characteristics are presented in table 1. Compared with Australian-born mothers; Chinese-born mothers were younger; experienced more socioeconomic disadvantage; a higher proportion were married; and a lower proportion were currently smoking. Birth weight was significantly lower in children of Chineseborn mothers and there were almost twice as many high birth weight children of Australian-born mothers. There were no significant differences between infants according to gestational age at birth, sex, Indigenous status nor birthing delivery method.

\section{Ethnic differences in growth trajectories}

The growth rates in each growth period of children of Chinese-born and Australian-born mothers living in Australia is presented in table 2. With adjustment for child birth weight, sex and socioeconomic disadvantage, the growth rate was significantly different at all time points between children of Chinese-born and Australianborn mothers except for 2-4 months and 25-44 months. Compared with children of Australian-born mothers, the growth rates for children of Chinese-born mothers were significantly higher between 0 and 2 months; significantly lower between 4 and 18 months and then significantly higher again between 18 and 25 months. There was no significant difference in growth rates between groups at age 2-4 months, nor 25-44 months.

The distinct differences in growth trajectories are represented in figure 1, whereby children of Chinese-born mothers experience a short deceleration at 0.5 months, then a sharp acceleration and period of rapid growth until 2 months. This period of accelerated growth occurs much earlier when compared with children of Australianborn mothers who do not begin accelerated growth until 2-4 months. The accelerated growth period of children of Chinese-born mothers is also steeper and spans across 1.5 months, whereby the accelerated growth period of children of Australian-born mothers is slower, spanning 2-18 months. Children of Chinese-born mothers have a higher predicted zBMI until $\sim 12$ months, subsequently children of Australian-born mothers have a higher predicted zBMI over time.

The predicted zBMI at each visit and the mean difference between Chinese versus Australian which constitute the growth curve are displayed in table 3 . The predicted mean zBMI is significantly different (either higher or lower) between children of Chinese-born and Australianborn mothers at all time points except for 12 months. The result at 12 months is consistent with the growth trajectory in figure 1, whereby the trajectories of the groups overlapped at this time point. Compared with children of 
Table 1 Demographic characteristics of Chinese-born and Australian-born mothers, their children living in Australia

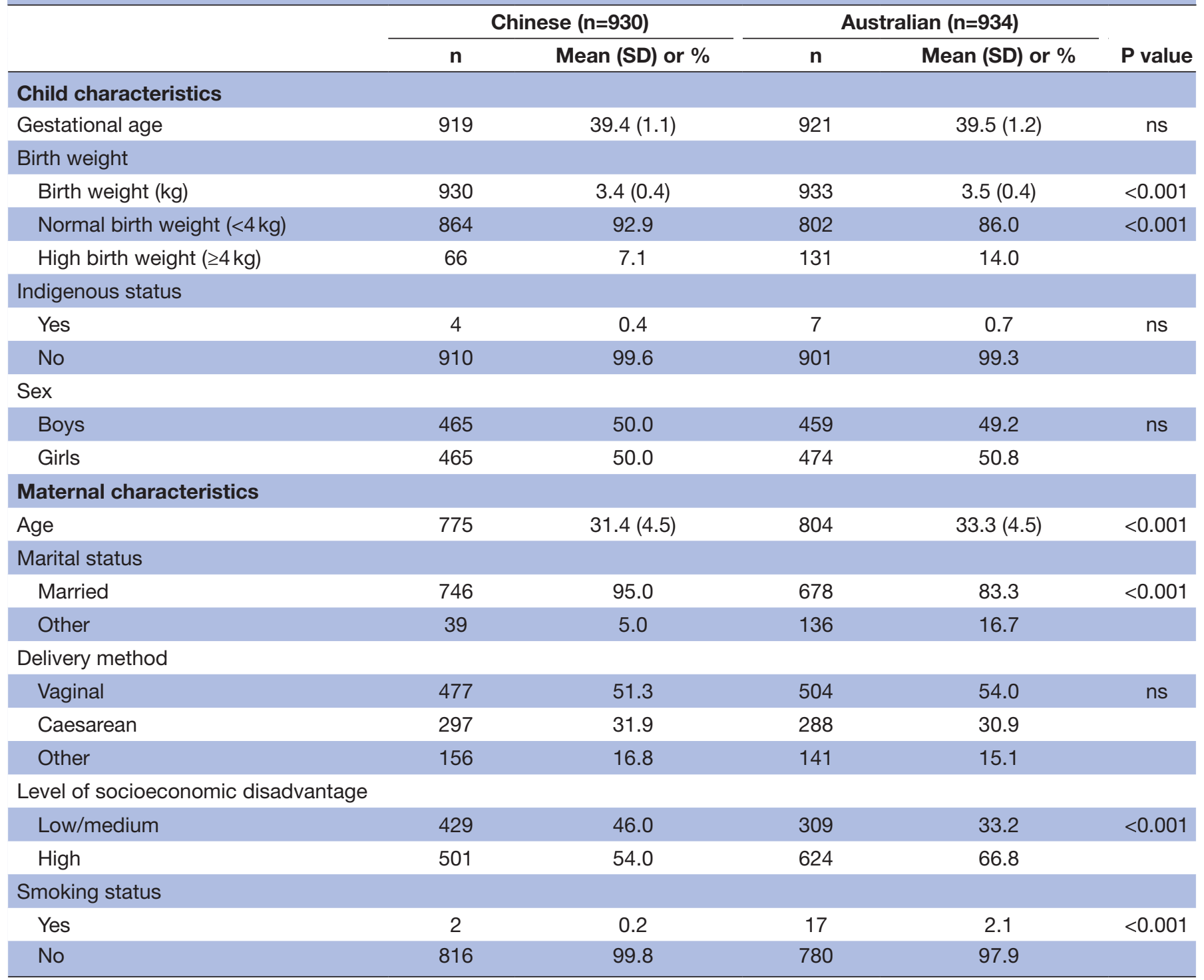

Level of disadvantage calculated using SEIFA and postcode.

ns, not significant; SEIFA, Socio-Economic Indexes for Areas.

Australian-born mothers, children of Chinese-born started with lower predicted zBMI from birth to 0.5 months, but then had a higher zBMI from 1 to 8 months. From 12 to 44 months children of Chinese-born mothers had a lower zBMI compared with their Australian counterparts.

\section{DISCUSSION}

This is the first known study to compare growth trajectories from birth to 3.5 years of age in children of Chineseborn compared with Australian-born mothers living in Victoria, Australia. This study reveals distinct ethnic differences in growth trajectories between children of Chinese-born compared with Australian-born mothers. In particular, children of Chinese-born mothers started with lower predicted zBMI from birth to 0.5 months, but then had a higher zBMI from 1 to 8 months and then from 12 to 44 months they had a lower predicted zBMI.
Early and sharp acceleration of growth was also observed for children of Chinese-born mothers (0.5-2 months) compared with a slower, longer acceleration of growth in children of Australian-born mothers (2-18 months).

There are a number of possible explanations for the ethnic disparities in growth reported in the current study. It is possible that the lower zBMI from birth to 0.5 months in children of Chinese-born immigrants is due to genetic factors. Anthropometric examination of 2695 full-term infants at birth in British Columbia, Canada revealed that Chinese and South Asian infants (ie, Indian, Pakistani, Bangladeshi) were smaller than Western counterparts. ${ }^{30}$ Recently, a narrative review also reported differences in foetal growth, birth weight, postnatal growth and body composition in Asian compared with Caucasian infants; and between Asian countries (eg, China, India, Hong Kong) and acknowledge the need to conduct longitudinal 
Table 2 Comparison of growth rates between children of Chinese-born and Australian-born mothers living in Australia from multilevel spline model

\begin{tabular}{|c|c|c|c|c|c|c|}
\hline \multirow[b]{2}{*}{ Period (months) } & \multicolumn{2}{|c|}{ Chinese } & \multicolumn{2}{|c|}{ Australian } & \multicolumn{2}{|c|}{ Chinese versus Australian } \\
\hline & Growth rate & $95 \% \mathrm{Cl}$ & Growth rate & $95 \% \mathrm{Cl}$ & Adjusted mean difference & $95 \% \mathrm{Cl}$ \\
\hline $0-0.5 \mathrm{~m}$ & -0.41 & -0.53 to -0.29 & -0.77 & -0.90 to -0.68 & $0.36^{*}$ & 0.19 to 0.54 \\
\hline $2-4 \mathrm{~m}$ & -0.0002 & -0.02 to 0.02 & 0.02 & 0.00 to 0.04 & -0.02 & -0.05 to 0.01 \\
\hline $4-18 \mathrm{~m}$ & -0.01 & -0.01 to 0.0001 & 0.06 & 0.06 to 0.06 & $-0.06^{\star}$ & -0.07 to -0.06 \\
\hline $25-44 m$ & 0.006 & 0.00 to 0.01 & 0.003 & 0.00 to 0.01 & 0.003 & 0.00 to 0.01 \\
\hline
\end{tabular}

Model adjusted for Indigenous status, child sex, gestational age, maternal marital status, delivery method, level of socioeconomic disadvantage (SEIFA), and smoking status. Values indicate the growth rate (slope) of the trajectory in each growth period (zBMI unit per month).

${ }^{*} \mathrm{P}<0.05$.

SEIFA, Socio-Economic Indexes for Areas; zBMI, body mass index z-score.

studies to understand more about influences on growth in the prepregnancy (eg, maternal weight), pregnancy (eg, diet, maternal weight gain) and postnatal (eg, feeding practices) stages. ${ }^{31}$

The reported differences in growth trajectory by ethnicity in this study may also question the suitability of applying the WHO growth reference charts for children of Chinese immigrants. For example, deviations from WHO growth standards have been reported for Hong Kong Chinese infants and Chinese infants who were shorter and lighter compared with WHO growth standards at different time points. ${ }^{31}$ Disparities from WHO growth standards have also been shown in adults (regardless of gender $)^{32}$ and school-aged children. ${ }^{33}$ Yang et al also ${ }^{34}$ reported differences in Chinese growth charts compared with WHO regarding undernutrition and obesity in a sample of children $(n=15886)$ indicating that differences could be due to sampling differences with children used to create the charts, differences in feeding criteria (ie,

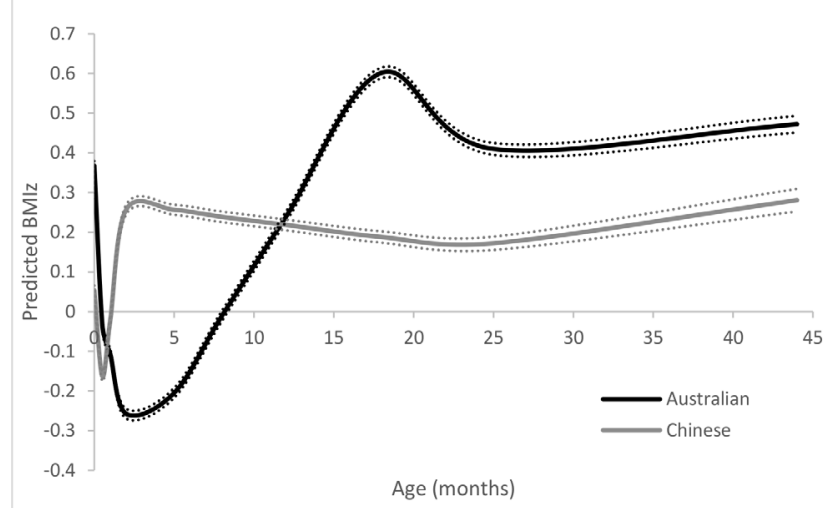

Figure 1 Average predicted zBMI trajectory by ethnicity from multilevel spline model with adjustment for covariates model adjusted for child sex, Indigenous status, gestational age, marital status, delivery method, smoking status, level of socioeconomic disadvantage. Dotted lines are 95\% Cl. zBMI, body mass index z-score mixed feeding in Chinese growth charts, exclusive breast feeding in WHO), and inclusion criteria for birth weight (low birth weight was excluded from Chinese growth charts and may change weight distribution). However, the WHO Multicentre Growth Reference Study found that variation in site (ie, country) only accounted for $3 \%$ of variation therefore race/genetic factors may not be the key factor driving differences in growth. ${ }^{34}$ Despite this, the WHO growth standards are considered valuable to promote healthy growth ${ }^{34}$ and these charts have been acknowledged as valuable for comparison among countries and regions. ${ }^{32}$ Future research to examine WHO growth standards and local growth charts to identify distinct differences among ethnicity ${ }^{34}$ and the implications for practice is required. ${ }^{32}$

The higher zBMI in children of Chinese-born mothers from 1 to 8 months may reflect cultural differences in feeding practices. Sociocultural factors, individual knowledge, beliefs and attitudes will influence a mother's infant feeding practices ${ }^{35}$ which will influence child growth. A common Chinese cultural belief is that a plump baby is a healthy baby ${ }^{3637}$ and social norms equate a heavy baby to high levels of parenting quality and competence. ${ }^{33}$ These cultural beliefs may encourage feeding practices that increase the risk of childhood overweight and obesity such as use of formula ${ }^{38}$ and non-responsive feeding practices. ${ }^{39}$ The Chinese-born immigrant infant feeding and growth hypothesis proposes that infant of Chineseborn mothers will be exposed to less breast feeding, more infant formula feeding and earlier introduction of solids-ultimately increasing protein intake. ${ }^{38}$ This higher protein intake in excess of requirements (Early Protein Hypothesis ${ }^{40}{ }^{41}$ may result in rapid growth trajectory and an increased risk of the infant being overweight and or obese ${ }^{38}$ Recent studies have highlighted the use of formula in this Chinese subgroup-with $90 \%$ of Chineseborn mothers introducing formula to their infants, with the average age of introduction of formula being 1 month of age; Chinese-born mothers being twice more likely to 
Table 3 Comparison of predicted zBMI between Australian versus Chinese children from multilevel spline model with adjustment for covariates

\begin{tabular}{|c|c|c|c|c|c|c|}
\hline & \multicolumn{2}{|c|}{ Chinese } & \multicolumn{2}{|c|}{ Australian } & \multicolumn{2}{|c|}{ Chinese versus Australian } \\
\hline & Mean zBMI & $95 \% \mathrm{Cl}$ & Mean zBMI & $95 \% \mathrm{Cl}$ & Adjusted mean difference & $95 \% \mathrm{Cl}$ \\
\hline Birth & 0.05 & 0.04 to 0.07 & 0.37 & 0.36 to 0.38 & $-0.31^{*}$ & -0.35 to -0.28 \\
\hline $0.5 \mathrm{~m}$ & -0.16 & -0.17 to -0.15 & -0.03 & -0.05 to -0.02 & $-0.13^{*}$ & -0.16 to -0.10 \\
\hline $1 \mathrm{~m}$ & -0.02 & -0.03 to -0.01 & -0.11 & -0.12 to -0.10 & $0.09^{*}$ & 0.05 to 0.12 \\
\hline $2 m$ & 0.26 & 0.25 to 0.27 & -0.26 & -0.27 to -0.25 & $0.51^{*}$ & 0.48 to 0.55 \\
\hline $5 \mathrm{~m}$ & 0.26 & 0.24 to 0.27 & -0.20 & -0.22 to -0.19 & $0.46^{*}$ & 0.43 to 0.49 \\
\hline $8 \mathrm{~m}$ & 0.24 & 0.23 to 0.25 & -0.01 & -0.02 to 0.00 & $0.25^{*}$ & 0.21 to 0.28 \\
\hline $12 \mathrm{~m}$ & 0.22 & 0.20 to 0.23 & 0.24 & 0.23 to 0.25 & -0.02 & -0.05 to 0.01 \\
\hline $18 \mathrm{~m}$ & 0.19 & 0.17 to 0.20 & 0.60 & 0.59 to 0.62 & $-0.41^{*}$ & -0.45 to -0.38 \\
\hline $25 \mathrm{~m}$ & 0.17 & 0.16 to 0.19 & 0.41 & 0.39 to 0.42 & $-0.24^{*}$ & -0.28 to -0.20 \\
\hline $44 \mathrm{~m}$ & 0.28 & 0.25 to 0.31 & 0.47 & 0.45 to 0.49 & $-0.19^{\star}$ & -0.26 to -0.13 \\
\hline
\end{tabular}

Model adjusted for Indigenous status, child sex, gestational age, birth weight, maternal marital status, delivery method, level of socioeconomic disadvantage (SEIFA) and smoking status.

${ }^{*} \mathrm{P}<0.05$.

m, month; SEIFA, Socio-Economic Indexes for Areas; zBMI, body mass index z-score.

use formula, and to introduce it earlier compared with Australian-born counterparts ${ }^{42}$; and more recently $55 \%$ of Chinese-born mothers to introduce formula in the first month of age (regardless of whether they breastfed). ${ }^{43}$

Breastfeeding, formula feeding and complementary feeding practices are complex to unpick and it can be difficult to isolate which feeding component is most influential on growth trajectory. ${ }^{44} \mathrm{~A}$ cross-sectional analysis of a national database revealed that disparities in early feeding practices exist in infants of Chinese-born compared with Australian-born mothers living in Australia. ${ }^{42}$ The key differences in feeding practices in infants of Chineseborn mothers include a higher proportion currently being breastfed; but of concern were obesity promoting behaviours such as being exposed to infant formula, water-based drinks (eg, cordial, soft drink) and fruit juice at a younger age. ${ }^{42}$

Furthermore, qualitative interviews with Chineseborn mothers have revealed the need to build support in feeding practices (ie, building confidence to breastfeed exclusively, dealing with grandparental pressure to formula feed, how to approach returning to work) and perceptions of healthy growth. ${ }^{35}$ The first 3 days post partum have been recently highlighted as a vulnerable period for formula supplementation; and breastfeeding control (mother's self-efficacy for breastfeeding) to predict exclusive breast feeding. Cultural understanding by health professionals of the influences on a mother's feeding practices and their effect on growth trajectory during infancy is required. For example, health professionals can play a role in supporting breast feeding intentions, self-efficacy and awareness of the Australian infant feeding guidelines. A recent qualitative study in 11 first time Chinese mothers in Australia also revealed the importance of integrating breast feeding with motherhood identity which motivated mothers and built self-efficacy in breast feeding, allowing greater persistence through breastfeeding challenges. ${ }^{45}$ Family members can also influence infant feeding practices in Chinese mothers. ${ }^{35}$ Culturally tailored strategies to support healthy growth which take into account cultural beliefs, attitudes, practices should be implemented by health professionals. This could include increasing access to face to face and online support from health professionals who are familiar with Chinese language and culture. ${ }^{35}$ It could also include strengthening family relationships and support for mothers throughout the perinatal period by educating spouses and grandmothers on breast feeding. ${ }^{45}$ Awareness of, and how to access support services such as lactation and mental health services is also required. ${ }^{45}$ Additional longitudinal research examining these factors, and the risk for developing overweight and obesity in this minority population is required.

The finding of a lower zBMI in children of Chineseborn mothers from 12 to 44 months compared with Australian counterparts could be due to cultural differences in diets. A recent study has revealed Chinese immigrants living in Australia eat significantly more vegetables and fruits per day; and less meat and cheese per week; compared with their Australian-born counterparts. ${ }^{46}$ Young children of Chinese immigrants in France have also been shown to consume significantly less dairy products compared with their French counterparts; along with eating less energy ( $\mathrm{kcal}$ ) per day at 1-3 years and 4-6 years old. ${ }^{47}$ Therefore, young children of Chinese heritage may have a diet composed of a higher proportion of vegetables and plant sources, rather than a typical Australian diet that tends to be higher in meat and protein and energy dense foods and beverages; and this may influence growth trajectory. 
Given that Asian populations have an increased risk of developing metabolic diseases at a lower BMI (due to the higher proportion of total and central adiposity compared with white populations) ${ }^{48}$ it is important for health professionals to track growth, feeding behaviours and other predictors (eg, level of disadvantage) over time to identify children who may be a risk of overweight and obesity later in life ${ }^{19}$ Monitoring of growth in early childhood is required to understand how children grow, what factors might explain differences in growth ${ }^{29}$ and what the risk of childhood overweight and obesity might be. It is also important to understand that ethnic minority groups are not homogeneous; and language, beliefs, heritage within particular ethnic groups need to be considered. ${ }^{49}$ The current study has highlighted early accelerated growth in Chinese-born mothers in this sample population. Rapid growth is a significant risk factor for later obesity ${ }^{9}$ therefore, a deeper understanding of the factors influencing growth patterns in these ethnic groups in order to intervene early is required. Longitudinal studies into later childhood and adulthood to track zBMI and related health outcomes long term is also recommended.

The strengths of this study include modelling growth trajectory in a large sample size with repeat measurements. ${ }^{29} 50$ The modelling approach (spline and knot methodology) has the strength of allowing examination of trajectories of zBMI across childhood while taking into account the different number of visits and measurements of children over time. ${ }^{50}$ This approach also allows the trajectories to be simplified, with a good fit between actual and predicted values ${ }^{50}$ and summarises the growth trajectories so they can be easily compared across populations. ${ }^{26}$ Another key strength of this study was the large sample size for specifically Chinese ethnic groups without having to aggregate this cultural group into 'Asians'; and obtain a deeper understanding of the specific ethnic disparities in growth. ${ }^{51}$ However, we also acknowledge several limitations. Children in this study were assigned to mother's self-reported ethnicity only ${ }^{29}$ and the father's self-reported ethnicity was largely missing. The repeat measurement data was drawn from one local government area in Victoria, which may not be generalisable to the wider population. ${ }^{29}$ Future studies should explore growth trajectories in a larger population drawn from the national population. Child anthropometry was measured objectively by different MCH nurses, however, $\mathrm{MCH}$ nurses are highly trained and follow consistent measurement procedures. Low birthweight infants were excluded, however, it has been suggested that universal low birth weight of $<2500 \mathrm{~g}$ may not be applicable to Asian children who are born with a lighter birth weight; and may overestimate the proportion classified as such. ${ }^{31}$ Other covariates such as maternal age could not be included in the model due the amount of missing data. Maternal BMI was not collected. While zBMI is a useful screening tool, assumptions about body composition and adiposity are limited using zBMI. Further research examining weight for age and length for age over time may also shed light on differences in growth trajectories. Data on child feeding measures (eg, breast feeding, formula feeding, mixed feeding and timing of the introductions of solids) would be beneficial to further explore the differences in growth patterns by ethnicity.

\section{CONCLUSION}

Ethnic disparities in growth trajectories between young children of Chinese-born compared with Australianborn mothers living in Victoria were revealed in models adjusted for birth weight, sex and level of socioeconomic disadvantage. A clearer understanding of these ethnically patterned growth trajectories is important for identifying key opportunities to promote healthy feeding and growth in early life in children of different ethnic groups, particularly for Chinese immigrants. Strategies to promote optimal growth will need to consider sociocultural factors. Further research is required to examine ethnic differences in growth into early childhood, and the risk of adiposity and other long-term health outcomes.

Acknowledgements We acknowledge and thank the City of Whitehorse, the custodian of the data, for granting access to the Maternal and Child Health data. Note the views in this paper do not necessarily represent those of the City of Whitehorse.

Contributors $\mathrm{KC}$ conceived the study. $\mathrm{KC}$ and $\mathrm{KAB}$ obtained access to the data. $\mathrm{KAB}$ supported data analysis and led the primary writing of the manuscript. $\mathrm{MZ}$ conducted the analysis of the data. $\mathrm{KAB}, \mathrm{PK}, \mathrm{RL}, \mathrm{KC}$ and $\mathrm{MZ}$ all contributed to interpretation of the findings and the development of the manuscript.

Funding MZ is supported by Australian National Health Medical Research Council Early Career Fellowship (GNT1124283). This work was supported by a School of Exercise and Nutrition Sciences (Deakin University) seeding grant.

Competing interests None declared.

Patient consent for publication Not required.

Ethics approval Approval for this study was provided by the Deakin University Human Research Ethics Committee (2014-184). The local government area who was the custodian of the database provided written permission for analysis of the fully deidentified data.

Provenance and peer review Not commissioned; externally peer reviewed.

Data availability statement Data may be obtained from a third party and are not publicly available. Deidentified data was obtained by specific request to a local government area of Victoria.

Supplemental material This content has been supplied by the author(s). It has not been vetted by BMJ Publishing Group Limited (BMJ) and may not have been peer-reviewed. Any opinions or recommendations discussed are solely those of the author(s) and are not endorsed by BMJ. BMJ disclaims all liability and responsibility arising from any reliance placed on the content. Where the content includes any translated material, BMJ does not warrant the accuracy and reliability of the translations (including but not limited to local regulations, clinical guidelines, terminology, drug names and drug dosages), and is not responsible for any error and/or omissions arising from translation and adaptation or otherwise.

Open access This is an open access article distributed in accordance with the Creative Commons Attribution Non Commercial (CC BY-NC 4.0) license, which permits others to distribute, remix, adapt, build upon this work non-commercially, and license their derivative works on different terms, provided the original work is properly cited, appropriate credit is given, any changes made indicated, and the use is non-commercial. See: http://creativecommons.org/licenses/by-nc/4.0/.

\section{ORCID iDs}

Kristy A Bolton http://orcid.org/0000-0001-6721-4503

Peter Kremer http://orcid.org/0000-0003-2476-1958 


\section{REFERENCES}

1 Sanders RH, Han A, Baker JS, et al. Childhood obesity and its physical and psychological co-morbidities: a systematic review of Australian children and adolescents. Eur J Pediatr 2015;174:715-46.

2 Simmonds M, Llewellyn A, Owen CG, et al. Predicting adult obesity from childhood obesity: a systematic review and meta-analysis. Obes Rev 2016;17:95-107.

3 Australian Bureau of Statistics. 4364055001D0016_20172018 National health survey: first results, 2017-18. Australia, Canberra: Australian Bureau of Statistics, ed, 2018.

4 Mameli C, Mazzantini S, Zuccotti GV. Nutrition in the first 1000 days: the origin of childhood obesity. Int J Environ Res Public Health 2016;13. doi:10.3390/ijerph13090838. [Epub ahead of print: 23 Aug 2016].

5 Woo Baidal JA, Locks LM, Cheng ER, et al. Risk factors for childhood obesity in the first 1,000 days: a systematic review. Am J Prev Med 2016;50:761-79.

6 Webster V, Denney-Wilson E, Knight J, et al. Describing the growth and rapid weight gain of urban Australian aboriginal infants. $J$ Paediatr Child Health 2013;49:303-8.

7 Adair LS. Child and adolescent obesity: epidemiology and developmental perspectives. Physiol Behav 2008;94:8-16.

8 Liu J, Liu J, Frongillo EA, et al. Body mass index trajectories during the first year of life and their determining factors. Am J Hum Biol 2019;31:e23188.

9 Zheng M, Bowe SJ, Hesketh KD, et al. Relative effects of postnatal rapid growth and maternal factors on early childhood growth trajectories. Paediatr Perinat Epidemiol 2019;33:172-80.

10 Australian Bureau of Statistics. 3412.0 - Migration, Australia, 201718: Commonwealth of Australia, 2019. Available: http://www.abs.gov. au/AUSSTATS/abs@.nsf/Lookup/3412.0Main+Features12017-18? OpenDocument [Accessed 28 Apr 2019].

11 Ke L, Brock KE, Cant RV, et al. The relationship between obesity and blood pressure differs by ethnicity in Sydney school children. Am J Hypertens 2009;22:52-8.

12 Waters E, Ashbolt R, Gibbs L, et al. Double disadvantage: the influence of ethnicity over socioeconomic position on childhood overweight and obesity: findings from an inner urban population of primary school children. Int J Pediatr Obes 2008;3:196-204.

13 Hardy LL, King L, Hector D, et al. Socio-cultural differences in Australian primary school children's weight and weight-related behaviours. J Paediatr Child Health 2013;49:641-8.

14 Scott B, Bolton KA, Strugnell C, et al. Weight status and obesityrelated dietary behaviours among culturally and linguistically diverse (CALD) children in Victoria, Australia. BMC Pediatr 2019;19:511.

15 State of Victoria (Department of Education and Training). Maternal and child health services, 2013. Available: http://www.education.vic. gov.au/childhood/parents/mch/Pages/about.aspx [Accessed 30 Mar 2015]

16 Baird J, Fisher D, Lucas P, et al. Being big or growing fast: systematic review of size and growth in infancy and later obesity. BMJ 2005;331:929.

17 Ong KK, Loos RJF. Rapid infancy weight gain and subsequent obesity: systematic reviews and hopeful suggestions. Acta Paediatr 2006;95:904-8.

18 Monteiro POA, Victora CG. Rapid growth in infancy and childhood and obesity in later life--a systematic review. Obes Rev 2005;6:143-54.

19 Bichteler A, Gershoff ET. Identification of children's BMI trajectories and prediction from weight gain in infancy. Obesity 2018;26:1050-6.

20 State Government of Victoria. Maternal and child health service, 2018. Available: https://www.education.vic.gov.au/parents/servicesfor-parents/Pages/mch.aspx [Accessed 30 Oct 2018].

21 Id the population experts. community profile, City of Whitehorse. Available: http://profile.id.com.au/whitehorse/birthplace [Accessed 25 Jun 2014]

22 Adhikari P, Cooper-Stanbury M. Australian National infant feeding survey, 2010 study documentation. Canberra: Australian Institute of Health and Welfare, 2014: 315.

23 Australian Bureau of Statistics. Socio-Economic indexes for areas: Commonwealth of Australia, 2018. Available: https://www.abs.gov. au/websitedbs/censushome.nsf/home/seifa [Accessed 06 Jan 2019].

24 World Health Organization. The WHO child growth standards, 2016. Available: http://www.who.int/childgrowth/en/ [Accessed 19 Sep 2016]

25 Australian Institute of Health and Welfare. Australia's children. Cat. no. CWS 69. Canberra: Australian Institute of Health and Welfare, 2020.

26 Howe LD, Tilling K, Matijasevich A, et al. Linear spline multileve models for summarising childhood growth trajectories: a guide to their application using examples from five birth cohorts. Stat Methods Med Res 2016;25:1854-74.

27 Zheng M, Cameron AJ, Birken CS, et al. Early infant feeding and BMI trajectories in the first 5 years of life. Obesity 2020;28:339-46.

28 Tilling K, Macdonald-Wallis C, Lawlor DA, et al. Modelling childhood growth using fractional polynomials and linear splines. Ann Nutr Metab 2014:65:129-38.

29 Fairley L, Petherick ES, Howe LD, et al. Describing differences in weight and length growth trajectories between white and Pakistani infants in the UK: analysis of the born in Bradford birth cohort study using multilevel linear spline models. Arch Dis Child 2013;98:274-9.

30 Janssen PA, Thiessen P, Klein MC, et al. Standards for the measurement of birth weight, length and head circumference at term in neonates of European, Chinese and South Asian ancestry. Open Med 2007;1:e74-88.

31 Muhardi L, Abrahamse-Berkeveld M, Acton D, et al. Differences in the anthropometry of Asian children and its role in metabolic health in later life: a narrative review. Obes Res Clin Pract 2016;10(Suppl 1):S3-16.

32 Zong $\mathrm{X}-\mathrm{N}$, Li H. Construction of a new growth references for China based on urban Chinese children: comparison with the WHO growth standards. PLoS One 2013;8:e59569.

33 Zhang $\mathrm{Y}-\mathrm{Q}$, Li $\mathrm{H}$, Wu H-H, et al. The 5 th national survey on the physical growth and development of children in the nine cities of China: Anthropometric measurements of Chinese children under 7 years in 2015. Am J Phys Anthropol 2017;163:497-509.

34 Yang Z, Duan Y, Ma G, et al. Comparison of the China growth charts with the WHO growth standards in assessing malnutrition of children. BMJ Open 2015;5:e006107.

35 Kuswara K, Laws R, Kremer P, et al. The infant feeding practices of Chinese immigrant mothers in Australia: a qualitative exploration. Appetite 2016;105:375-84.

36 Diong S, Johnson M, Langdon R. Breastfeeding and Chinese mothers living in Australia. Breastfeed Rev 2000;8:17-23.

37 Chen S, Binns CW, Maycock B, et al. Chinese mothers' perceptions of their child's weight and obesity status. Asia Pac J Clin Nutr 2014;23:452-8.

38 Bolton KA, Kremer P, Hesketh KD, et al. The Chinese-born immigrant infant feeding and growth hypothesis. BMC Public Health 2016;16:1071.

39 Ventura AK. Associations between breastfeeding and maternal responsiveness: a systematic review of the literature. Adv Nutr 2017;8:495-510.

40 Koletzko B, von Kries R, Closa R, et al. Can infant feeding choices modulate later obesity risk? Am J Clin Nutr 2009;89:1502S-8.

41 Koletzko B, Broekaert I, Demmelmair H, et al. Protein intake in the first year of life: a risk factor for later obesity? The E.U. childhood obesity project. Adv Exp Med Biol 2005;569:69-79.

42 Bolton KA, Kremer P, Hesketh KD, et al. Differences in infant feeding practices between Chinese-born and Australian-born mothers living in Australia: a cross-sectional study. BMC Pediatr 2018;18:209.

43 Kuswara K, Campbell KJ, Hesketh KD, et al. Patterns and predictors of exclusive breastfeeding in Chinese Australian mothers: a cross sectional study. Int Breastfeed J 2020;15:61.

44 Ejlerskov KT, Christensen LB, Ritz C, et al. The impact of early growth patterns and infant feeding on body composition at 3 years of age. Br J Nutr 2015;114:316-27.

45 Kuswara K, Knight T, Campbell KJ, et al. Breastfeeding and emerging motherhood identity: an interpretative phenomenological analysis of first time Chinese Australian mothers' breastfeeding experiences. Women Birth 2020;387. doi:10.1016/j.wombi.2020.03.005. [Epub ahead of print: 20 Mar 2020].

46 Astell-Burt T, Feng X, Croteau K, et al. Influence of neighbourhood ethnic density, diet and physical activity on ethnic differences in weight status: a study of 214,807 adults in Australia. Soc Sci Med 2013;93:70-7.

47 Rovillé-Sausse FN. Westernization of the nutritional pattern of Chinese children living in France. Public Health 2005;119:726-33.

48 Ramachandran A, Chamukuttan S, Shetty SA, et al. Obesity in Asia--is it different from rest of the world. Diabetes Metab Res Rev 2012;28(Suppl 2):47-51.

49 Patel R, Tilling K, Lawlor DA, et al. Socioeconomic differences in childhood length/height trajectories in a middle-income country: a cohort study. BMC Public Health 2014;14:932.

50 Howe LD, Tilling K, Galobardes B, et al. Socioeconomic differences in childhood growth trajectories: at what age do height inequalities emerge? J Epidemiol Community Health 2012;66:143-8.

51 Alcalá HE, Sharif MZ. Going flat: examining heterogeneity in the soda-obesity relationship by subgroup and place of birth among Asian Americans. Public Health Nutr 2017;20:1380-7. 